\title{
Predictive Value of QRS Duration at Admission for In-Hospital Clinical Outcome of Takotsubo Cardiomyopathy
}

Tetsuo Yamaguchi, MD; Tsutomu Yoshikawa, MD; Toshiaki Isogai, MD;

Takamichi Miyamoto, MD; Yuichiro Maekawa, MD; Tetsuro Ueda, MD;

Konomi Sakata, MD; Tsutomu Murakami, MD; Takeshi Yamamoto, MD;

Ken Nagao, MD; Morimasa Takayama, MD

Background: Prolonged QRS duration (pQRSd) on electrocardiogram (ECG) is a strong predictor of poor outcome in heart failure, myocardial infarction, and myocarditis, but it is unclear whether pQRSd also predicts poor outcomes of takotsubo cardiomyopathy (TC).

Methods and Results: Between 1 January 2010 and 31 December 2012, we retrospectively enrolled 299 patients with TC (mean age, $73.5 \pm 11.7$ years; $21.4 \%$ male) from the Tokyo CCU Network database, which consists of 71 cardiovascular centers in the metropolitan area. In-hospital clinical outcomes were compared between patients with pQRSd on admission ECG (QRS $\geq 120 \mathrm{ms;}$ $\mathrm{n}=34$ ) and those with normal QRS duration ( $<120 \mathrm{~ms} ; \mathrm{n}=265)$. The in-hospital mortality rate for pQRSd was significantly higher than that for normal QRS duration ( $23.5 \%$ vs. $3.8 \%, P<0.001)$. Similarly, prevalence of ventilator use $(38.2 \%$ vs. $11.4 \%, P<0.001)$, ventricular tachycardia or fibrillation $(14.7 \%$ vs. $1.5 \%, \mathrm{P}<0.001)$, and circulatory failure requiring catecholamine or cardiopulmonary supportive devices $(41.2 \%$ vs. $14.0 \%, P<0.001)$ was significantly higher in the pQRSd group. On multivariate logistic regression analysis, pQRSd was an independent predictor for both in-hospital mortality (OR, 5.06; 95\% $\mathrm{Cl}$ : 1.79-14.30, $\mathrm{P}=0.002)$ and cardiac death (OR, 7.34; 95\% Cl: 1.33-40.51, $\mathrm{P}=0.02)$.

Conclusions: TC with pQRSd is associated with poor in-hospital clinical outcome. Aggressive intervention may be required to prevent severe complications in these patients.

Key Words: Electrocardiography; Prolonged QRS duration; Takotsubo cardiomyopathy

$\mathbf{T}$ akotsubo cardiomyopathy (TC) is characterized by reversible left ventricular (LV) dysfunction not caused by coronary artery disease (CAD). ${ }^{1-3}$ The in-hospital mortality rate is between $0 \%$ and $8 \%,{ }^{2-5}$ and the rate of major adverse cardiac and cerebrovascular events is 7.1\%. ${ }^{5} \mathrm{~J}$ wave and QT prolongation on electrocardiogram (ECG) are predictors of short-term outcome in TC,, 7 but the prognostic impact of prolonged QRS duration (pQRSd) in TC remains unknown, although it is a strong predictor of poor outcome in previous myocardial infarction, congestive heart failure (HF), and myocarditis. ${ }^{8-10}$

The aim of the present study was to examine the predictive value of $\mathrm{pQRSd}$ for in-hospital clinical outcomes of $\mathrm{TC}$, using a multicenter database in Japan.

\section{Methods}

Subjects

The Tokyo CCU Network database is a prospective, ongo- ing, multicenter, population-based cohort database of emergency admissions to acute cardiac care facilities in Tokyo. It was launched in 1978 and expanded to 71 largevolume cardiovascular facilities. We launched the TC research group in 2010 and started collecting data on patients with TC admitted to facilities participating in the Tokyo CCU Network. Data on TC patients were collected retrospectively, and included original copies of ECG for a 3-year period between 2010 and 2012. Data have been collected prospectively since 2013 , but do not include original copies of ECG. Therefore, we used data collected between 2010 and 2012 for this study. The requirement for informed consent was waived because all data were anonymously catalogued. As stated in a preceding article from a Tokyo CCU Network registered cohort, ${ }^{11}$ patient data collection policies are displayed at the emergency entrance of each facility in accordance with rules set forth by the Ministry of Internal Affairs and Communications. The aim of the registry and personal information protection policy

Received September 7, 2016; revised manuscript received October 24, 2016; accepted October 31, 2016; released online December 2, 2016 Time for primary review: 14 days

Tokyo CCU Network Scientific Committee, Tokyo (T. Yamaguchi, T. Yoshikawa, T.I., Y.M., T.U., K.S., T. Murakami, T. Yamamoto, K.N., M.T.); Department of Cardiology, Japanese Red Cross Musashino Hospital, Tokyo (T. Yamaguchi, T. Miyamoto), Japan

Mailing address: Tetsuo Yamaguchi, MD, Department of Cardiology, Japanese Red Cross Musashino Hospital, 1-26-1 Kyonanncho, Musashino, Tokyo 180-8610, Japan. E-mail: tetsuo@musashino.jrc.or.jp

ISSN-1346-9843 All rights are reserved to the Japanese Circulation Society. For permissions, please e-mail: cj@j-circ.or.jp 
are stated on pamphlets given to the patients and their family. Trained personnel collected data via individual chart review. The quality of submitted data was maintained through annual data quality checks that included point-ofentry mechanism controls. ${ }^{11}$ This study was approved by the institutional review board of Tokyo CCU Network Scientific Committee and conformed to the ethical principles of the Declaration of Helsinki.

We first identified 368 consecutive patients registered as having TC from all the facilities that participated in the Tokyo CCU Network between 1 January 2010 and 31 December 2012. After excluding patients who did not undergo coronary angiography $(n=56)$ and those without ECG information ( $n=13)$, we finally enrolled 299 eligible patients (Figure 1). We defined TC according to the criteria proposed by the Mayo Clinic, ${ }^{12}$ as the presence of transient LV wall motion abnormality beyond a single epicardial coronary artery perfusion territory, the absence of obstructive CAD or angiographic evidence of acute plaque rupture, the presence of new ECG abnormalities (either ST-segment elevation and/or T-wave inversion) or elevation in cardiac troponin levels, and the absence of pheochromocytoma and myocarditis.

\section{Data Collection}

The following data were collected using a Tokyo CCU Network database questionnaire: age, sex, height, weight, initial vital signs (including systolic blood pressure [SBP] and diastolic blood pressure, heart rate, and arterial oxygen saturation in the emergency room), Killip classification, ${ }^{\mathbf{1 3}}$ ECG findings at admission (ST-segment elevation and depression, QT interval, and QRS duration), laboratory data at admission, echocardiographic parameters (LV

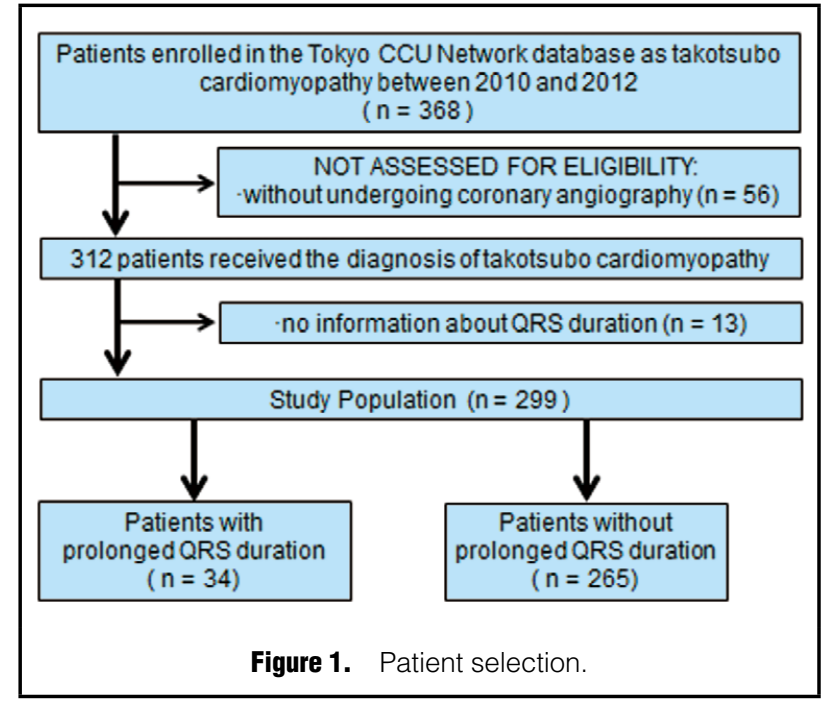

ejection fraction $[E F]$ by a modified Simpson method, and LV outflow tract obstruction), and cardiac catheterization findings. Shock on arrival was defined as SBP in the emergency room $<90 \mathrm{mmHg}$. HF on arrival was defined as Killip class $\geq$ II at admission. QT prolongation was defined as QTc (Bazett's formula) $\geq 440 \mathrm{~ms}$, and QRS prolongation was defined as $Q R S \geq 120 \mathrm{~ms}$. We initially collected original copies of ECG at admission in 2010 and at both admission and discharge in 2011 and 2012. Then, we collected original copies of the ECG at discharge in patients who had been admitted with pQRSd in 2010. Estimated glomerular fil-

\section{Table 1. Baseline Subject Characteristics}

\begin{tabular}{|c|c|c|c|}
\hline & $\begin{array}{c}\text { pQRSd } \\
(n=34)\end{array}$ & $\begin{array}{c}\text { Normal QRSd } \\
\quad(n=265)\end{array}$ & P-value \\
\hline Age (years) & $69.8 \pm 12.5$ & $74.0 \pm 11.6$ & 0.021 \\
\hline Sex: male & $10(29.4)$ & $54(20.4)$ & 0.23 \\
\hline $\mathrm{BMI}\left(\mathrm{kg} / \mathrm{m}^{2}\right)$ & $20.8 \pm 4.4$ & $20.6 \pm 3.6$ & 0.71 \\
\hline $\mathrm{SBP}(\mathrm{mmHg})(\mathrm{n}=294)$ & $130 \pm 42(n=33)$ & $136 \pm 32(n=261)$ & 0.39 \\
\hline Heart rate (beats $/ \mathrm{min})(n=293)$ & $103 \pm 36(n=32)$ & $88 \pm 24(n=262)$ & 0.033 \\
\hline ST-segment elevation $(n=295)$ & $25(73.5)$ & $200(76.6)(n=261)$ & 0.69 \\
\hline QT prolongation $(\mathrm{n}=298)$ & $24(70.6)$ & $113(42.8)(n=264)$ & 0.002 \\
\hline $\operatorname{LVEF}(\%)^{\dagger}(n=185)$ & $49.3 \pm 14.7(n=21)$ & $50.7 \pm 13.6(n=164)$ & 0.63 \\
\hline LVOT (n=262) & $3(11)(n=28)$ & $21(9.0)(n=234)$ & 0.76 \\
\hline Apical ballooning $(n=292)$ & $32(94.1)$ & $226(87.6)(n=258)$ & 0.27 \\
\hline Emotional stress $(n=282)$ & $11(33.3)(n=33)$ & $72(28.9)(n=249)$ & 0.68 \\
\hline Physical stress $(\mathrm{n}=282)$ & $17(51.5)(n=33)$ & $87(34.9)(n=249)$ & 0.088 \\
\hline HF on arrival $(n=284)$ & $12(36.4)(n=33)$ & $48(19.1)(n=251)$ & 0.023 \\
\hline eGFR $\left(\mathrm{mL} / \mathrm{min} / 1.73 \mathrm{~m}^{2}\right)(\mathrm{n}=289)$ & $41.7(34.1-51.96)$ & $35.5(30.4-47.9)(n=255)$ & 0.095 \\
\hline Creatinine kinase $(I U / L)(n=274)$ & $214(132.3-303.3)(n=30)$ & $167(101.0-335.0)(n=244)$ & 0.012 \\
\hline$B N P \times U L N(n=275)$ & $18.77(6.65-32.73)(n=31)$ & $7.96(3.18-23.61)(n=244)$ & $<0.001$ \\
\hline WBC $(/ \mu \mathrm{L})(\mathrm{n}=298)$ & $8,900(7,500-12,000)(n=33)$ & $7,900(6,030-10,800)$ & 0.27 \\
\hline $\operatorname{CRP}(\mathrm{mg} / \mathrm{dL})(\mathrm{n}=294)$ & $0.46(0.1-2.9)(n=33)$ & $0.28(0.1-1.5)(n=261)$ & 0.21 \\
\hline Positive troponin $(n=257)$ & $21(87.5)(n=24)$ & $195(83.7)(n=233)$ & 0.63 \\
\hline
\end{tabular}

Data given as mean \pm SD, $n(\%)$ or median (IQR). ${ }^{\dagger}$ Modified Simpson method. Heart failure on arrival was defined as Killip class $\geq 1 /$ at admission. BNP and the N-terminal of prohormone brain natriuretic peptide are described as the ratio of the ULN. BMI, body mass index; BNP, brain natriuretic peptide; CRP, C-reactive protein; eGFR, estimated glomerular filtration rate; HF, heart failure; LVEF, left ventricular ejection fraction; LVOT, left ventricular outflow tract obstruction; pQRSd, prolonged QRS duration; QRSd, QRS duration; SBP, systolic blood pressure; ULN, upper limit of normal range; WBC, white blood cells. 


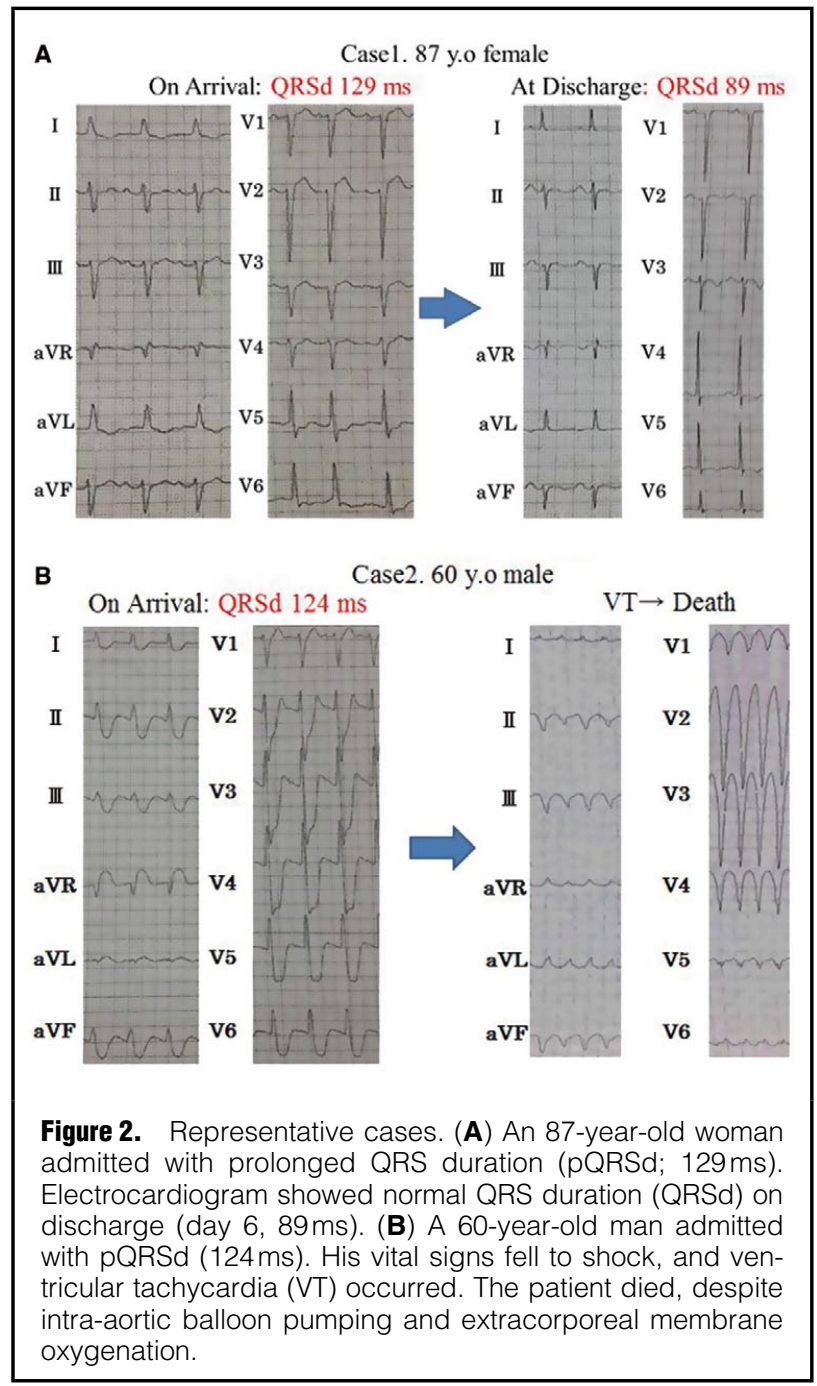

tration rate (eGFR; $\mathrm{mL} / \mathrm{min} / 1.73 \mathrm{~m}^{2}$ ) was calculated as $194 \times \mathrm{Cr}-1.094 \times$ age -0.287 in men, and as $194 \times \mathrm{Cr}-1.094 \times$ age- $0.287 \times 0.739$ in women. ${ }^{14}$ Brain natriuretic peptide (BNP) and the N-terminal of prohormone BNP were converted to the ratio of the upper limit of the normal range.
We recorded all in-hospital complications, including allcause death, definite cardiac death (defined as death from HF and arrhythmia), ventricular arrhythmia (ventricular tachycardia or ventricular fibrillation), advanced atrioventricular block (Mobitz type II atrioventricular block or complete atrioventricular block); use of catecholamine, invasive or non-invasive ventilator, cardiopulmonary supportive device (intra-aortic balloon pumping [IABP] or extracorporeal membrane oxygenation); and circulatory failure requiring catecholamine or a cardiopulmonary supportive device.

\section{Statistical Analysis}

Continuous variables are expressed as mean \pm SD if the variable was normally distributed, or as median (IQR) if not. Categorical variables are expressed as n (\%). We used Welch's t-test or Wilcoxon's rank-sum test to compare continuous variables, and Fisher's exact test to compare categorical variables. Univariate logistic regression analysis was used to identify univariate predictors for in-hospital mortality. The selection of variables for univariate analysis was based on clinical importance and data from preceding studies. Only variables that had 2 -sided $\mathrm{P}<0.2$ on univariate logistic regression analyses were included in multivariate logistic regression analysis to determine independent predictors for in-hospital mortality. We performed a complete case analysis for the multivariate logistic regression analysis. Receiver operating characteristic (ROC) analysis was performed to evaluate sensitivity and specificity of QRS duration for outcome. Statistical analysis was performed using R (version 3.1.0; R Development Core Team). Two-sided $\mathrm{P}<0.05$ was considered statistically significant.

\section{Results}

\section{Patient Characteristics}

As shown in Table 1, 34 patients (11.4\%) had pQRSd, of whom $7(20.6 \%)$ had right bundle branch block, $13(38.2 \%)$ had left bundle branch block, and $14(41.2 \%)$ had nonspecific intraventricular block. Patients with $\mathrm{pQRSd}$ had a significantly higher heart rate, higher creatinine kinase, eGFR, BNP, and higher prevalence of QT prolongation and of HF on arrival. In contrast, there were no significant differences in sex, SBP, LVEF, apical ballooning, positive troponin, or preceding stress.

\begin{tabular}{|c|c|c|c|}
\hline & $\begin{array}{l}\text { pQRSd } \\
(\mathrm{n}=34)\end{array}$ & $\begin{array}{l}\text { Normal QRSd } \\
(n=265)\end{array}$ & P-value \\
\hline All-cause death & $8(23.5)$ & $10(3.8)$ & $<0.001$ \\
\hline Cardiac death & $4(11.8)$ & $4(1.5)$ & $<0.001$ \\
\hline Ventricular arrhythmia ${ }^{\dagger}$ & $5(14.7)$ & $4(1.5)$ & $<0.001$ \\
\hline Advanced atrioventricular block $\ddagger$ & $4(11.8)$ & $10(3.4)$ & 0.024 \\
\hline Catecholamines & $8(23.5)$ & $28(10.7)$ & 0.031 \\
\hline Cardiopulmonary supportive device $\S$ & $6(17.6)$ & $9(3.4)$ & $<0.001$ \\
\hline Circulatory failure $^{\dagger \dagger}$ & $14(41.2)$ & $37(14.0)$ & $<0.001$ \\
\hline Ventilatorn & $13(38.2)$ & $30(11.4)$ & $<0.001$ \\
\hline
\end{tabular}

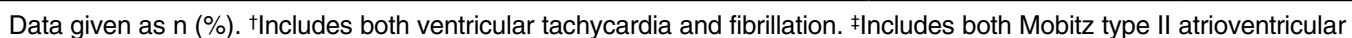
block and complete atrioventricular block. \$Intra-aortic balloon pumping and/or extracorporeal membrane oxygenation. t+Defined as use of catecholamine or cardiopulmonary supportive devices. INon-invasive positive pressure support and mechanical ventilation. pQRSd, prolonged QRS duration; QRSd, QRS duration. 


\begin{tabular}{|c|c|c|c|c|}
\hline \multirow{2}{*}{ Variables } & \multicolumn{2}{|c|}{ Univariate } & \multicolumn{2}{|c|}{ Multivariate (total, $n=262$ ) } \\
\hline & OR $(95 \% \mathrm{Cl})$ & P-value & OR $(95 \% \mathrm{Cl})$ & P-value \\
\hline Age & $0.99(0.96-1.03)$ & 0.60 & NA & NA \\
\hline Male sex & $0.78(0.23-2.70)$ & 0.70 & NA & NA \\
\hline Shock on arrival & $5.63(2.10-15.1)$ & $<0.001$ & $4.32(1.39-13.45)$ & 0.011 \\
\hline HF on arrival & $3.26(1.30-8.15)$ & 0.011 & $3.21(1.17-8.80)$ & 0.020 \\
\hline pQRSd & $6.61(2.62-16.7)$ & $<0.001$ & $5.06(1.79-14.30)$ & 0.002 \\
\hline pQTd & $1.02(0.75-1.38)$ & 0.90 & NA & NA \\
\hline Emotional stress & $0.78(0.25-2.52)$ & 0.69 & NA & NA \\
\hline Physical stress & $1.36(0.49-3.75)$ & 0.56 & NA & NA \\
\hline Heart rate & $1.02(1.00-1.03)$ & 0.020 & $1.01(0.99-1.03)$ & 0.26 \\
\hline WBC count & $1.00(1.00-1.01)$ & 0.079 & $1.00(1.00-1.00)$ & 0.18 \\
\hline BNP & $1.02(0.99-1.04)$ & 0.14 & $1.01(0.98-1.04)$ & 0.51 \\
\hline
\end{tabular}

NA, not applicable; pQTd, prolonged QT duration. Other abbreviations as in Table 1.

\begin{tabular}{|c|c|c|c|c|}
\hline \multirow{2}{*}{ Variables } & \multicolumn{2}{|c|}{ Univariate } & \multicolumn{2}{|c|}{ Multivariate (total, $n=262$ ) } \\
\hline & OR (95\% Cl) & P-value & OR (95\% Cl) & P-value \\
\hline Age & $0.97(0.92-1.02)$ & 0.26 & NA & NA \\
\hline Male sex & $2.25(0.52-9.69)$ & 0.27 & NA & NA \\
\hline Shock on arrival & $28.13(6.17-128.3)$ & $<0.001$ & $24.62(4.53-133.68)$ & $<0.001$ \\
\hline HF on arrival & $4.87(1.13-20.95)$ & 0.031 & $2.05(0.38-11.06)$ & 0.41 \\
\hline pQRSd & $8.70(2.07-36.9)$ & 0.003 & $7.34(1.33-40.51)$ & 0.023 \\
\hline pQTd & $2.25(0.45-11.36)$ & 0.32 & NA & NA \\
\hline Emotional stress & $1.83(0.40-8.35)$ & 0.44 & NA & NA \\
\hline Physical stress & $2.33(0.51-10.64)$ & 0.27 & NA & NA \\
\hline Heart rate & $1.02(1.00-1.04)$ & 0.002 & $1.02(0.99-1.06)$ & 0.10 \\
\hline WBC count & $1.00(0.99-1.00)$ & 0.30 & NA & NA \\
\hline BNP & $1.02(0.99-1.05)$ & 0.12 & $1.03(0.99-1.07)$ & 0.21 \\
\hline
\end{tabular}

Abbreviations as in Tables 1,3.

\section{Representative Cases}

In the present study, there were 2 representative cases of pQRSd. The first patient (Figure 2A) was an 87-year-old woman who was admitted with pQRSd (129 ms). During hospitalization, she developed HF but recovered in a few days. ECG showed normal QRS duration on discharge (day 6,89ms). The second patient (Figure 2B) was a 60-yearold man who was admitted with pQRSd $(124 \mathrm{~ms})$ and STsegment depression. After admission, his vital signs fell, and shock and ventricular tachycardia occurred. QRS duration was not normalized. Despite undergoing IABP insertion and extracorporeal membrane oxygenation, he died. In this study, 5 patients similarly had persistent $\mathrm{pQRSd}$, and 4 of them died.

\section{Clinical Outcomes}

Overall in-hospital mortality was $6.0 \%(18 / 299)$, of which definite cardiac death comprised $44.4 \%(8 / 18)$. The other causes of death were documented as pneumonia $(n=4)$, sepsis $(n=2)$, stroke or brain hemorrhage $(n=2)$, and malignancy $(\mathrm{n}=2)$. The in-hospital mortality was significantly higher in the $\mathrm{pQRSd}$ group than in those with normal QRS duration $(23.5 \%$ vs. $3.8 \%, \mathrm{P}<0.001$; Table 2). Similarly, definite cardiac death was more frequent in the pQRSd than in those with normal QRS duration $(11.8 \%$ vs. $1.5 \%$,
$\mathrm{P}<0.001$; Table 2). In addition, the prevalence of other clinical outcomes, including ventricular arrhythmia, advanced atrioventricular block, ventilator use, use of cardiopulmonary supportive device, and circulatory failure, was significantly higher in the $\mathrm{pQRSd}$ group (Table 2). On univariate logistic regression analysis, shock on arrival, HF on arrival, $\mathrm{pQRSd}$, heart rate, white blood cell count, and BNP level had a 2 -sided $\mathrm{P}<0.2$ for in-hospital mortality. On multivariate logistic regression analysis, pQRSd (OR, 5.06; 95\% CI: 1.79-14.30, $\mathrm{P}=0.002)$, as well as shock on arrival (OR, 4.32, 95\% CI: $1.39-13.45, \mathrm{P}=0.011)$ and $\mathrm{HF}$ on arrival (OR, 3.21, 95\% CI: $1.17-8.80, \mathrm{P}=0.020$ ) were significantly associated with in-hospital mortality (Table 3). Likewise, another multivariate logistic regression analysis showed that $\mathrm{pQRSd}$ was significantly associated with cardiac death (OR, 7.34, 95\% CI: 1.33-40.51, P=0.023; Table 4). On ROC analysis, QRS duration had high predictive value for both in-hospital death (area under the curve [AUC], 0.859) and cardiac death (AUC, 0.901; Figure 3). For in-hospital death, with the optimal cut-off of $110 \mathrm{~ms}$, the sensitivity and specificity were 0.722 and 0.854 , respectively; and with a cut-off of $120 \mathrm{~ms}$ (definition of pQRSd), the sensitivity and specificity were 0.444 and 0.907 , respectively. For cardiac death, with the optimal cut-off of $110 \mathrm{~ms}$, the sensitivity and specificity were 0.838 and 0.875 , respectively; and with the cut- 

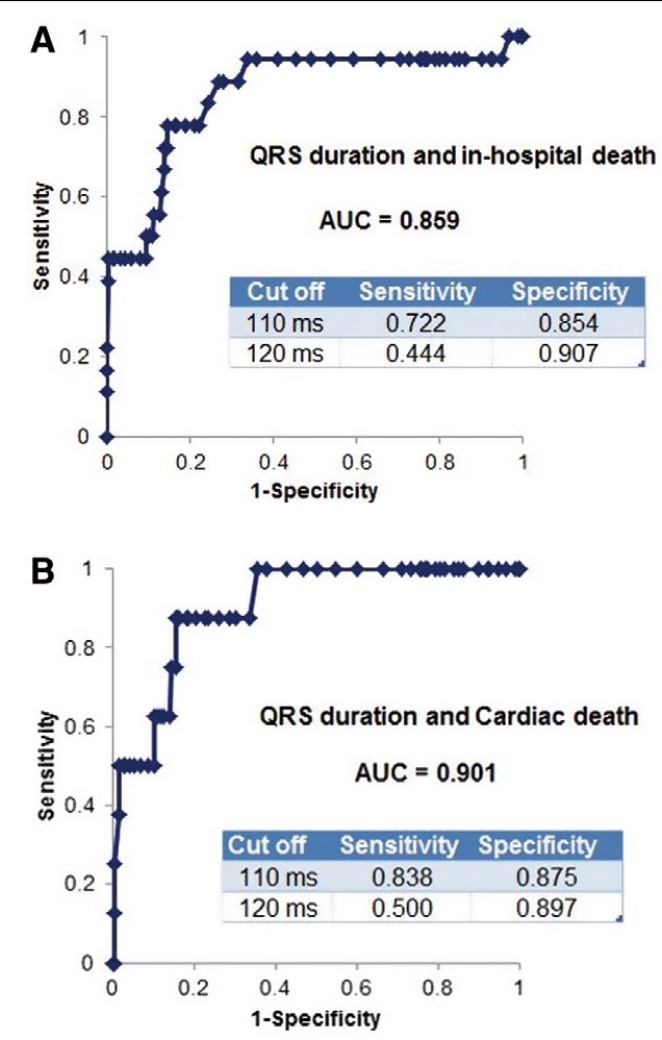

Figure 3. Receiver operating characteristic curves of QRS duration for $(\mathbf{A})$ in-hospital mortality and (B) cardiac death. $A \cup C$, area under the curve.

off of $120 \mathrm{~ms}$, the sensitivity and specificity were 0.500 and 0.897 , respectively.

\section{Normalization of pQRSd at Discharge}

The QRS duration was normalized at discharge in 29 $(85.3 \%)$ of 34 patients with $\mathrm{pQRSd}$. Four of 5 patients with persistent $\mathrm{pQRSd}$ died.

\section{Discussion}

In the present study, we compared the clinical outcomes of TC with and without $\mathrm{pQRSd}$, using data from a large, multicenter registry. The main findings were that $\mathrm{pQRSd}$ at admission was independently associated with in-hospital death and cardiac death. In addition, critical complications, including ventricular arrhythmia, use of a ventilator, and circulatory failure, were more frequently found in patients with pQRSd.

In this study, 18 fatal events were observed during hospitalization, of which only 8 were definite cardiac deaths. We speculated that the other 10 deaths may be attributable to both severe physical stresses (such as infection, cerebrovascular disease, and malignancy) and TC, because TC is often caused by physical stress $(34.8 \%$ of the present patients had existing physical stress), and the combination of the aforementioned diseases was reported to be significantly associated with higher in-hospital mortality. ${ }^{15}$

$\mathrm{J}$ wave and QT prolongation on ECG are predictors of short-term TC outcome, ${ }^{6,7}$ but QT prolongation was not an independent predictor for in-hospital mortality in this study. The difference in results may be explained by the difference in patient background. In the present study, we analyzed all patients hospitalized with TC, but the previous report analyzed only those with arrhythmia. ${ }^{7}$ In contrast, no previous study has investigated the frequency and prognostic impact of pQRSd in TC. In this study, pQRSd was noted in $11.4 \%$ of patients (34/299). We also showed that pQRSd was associated with poor outcome. This may be biologically plausible, because pQRSd suggests electrical stunning of the myocardium or Purkinje-ventricular conduction delay caused by myocardial ischemia. ${ }^{\mathbf{1 6}, 17}$

pQRSd is significantly associated with poor outcome in a variety of diseases, including previous myocardial infarction, congestive HF, and myocarditis. ${ }^{8-10}$ In ischemic cardiomyopathy, ischemia causes leakage of intracellular potassium to the extracellular space, which increases extracellular potassium concentration. High extracellular potassium concentration delays electrical conduction of the myocardium, leading to $\mathrm{pQRSd}$ and myocardial dysfunction. 18,19 In myocarditis, inflammation causes degeneration and necrosis of the myocardial conduction cells and interstitial edema, which is thought to play a major role in the development of pQRSd. ${ }^{20}$ In addition, pQRSd has been reported as a predictor of fulminant myocarditis. ${ }^{21}$ In contrast, in TC, the cause and clinical significance of pQRSd remains unclear. The QRS duration of most of the present patients $(29 / 34,85.3 \%)$ was normalized at discharge, suggesting that $\mathrm{pQRSd}$ in TC is an exclusively transient phenomenon, similar to myocarditis. Although there were no significant differences, creatinine kinase, BNP, white blood cell count, and C-reactive protein tended to be higher in the $\mathrm{pQRSd}$ group than in the normal QRS group (Table 1). This suggests that severe myocardial inflammation might occur in patients with $\mathrm{pQRSd}$. Only one case report noted transient $\mathrm{pQRSd}$ in TC.22 In that report, the patient had pulmonary edema and $\mathrm{pQRSd}$ on admission, but the QRS duration was normalized immediately after the introduction of IABP. This may indicate that pQRSd in TC reflects myocardial ischemia due to microvascular dysfunction, because IABP improves coronary driving pressure. In addition, improvement of hemodynamics by IABP seemed to improve acidosis, which might contribute to the normalization of QRS duration. In summary, we assume that $\mathrm{pQRSd}$ in TC is the result of transient inflammation of the myocardium or myocardial ischemia due to microvascular dysfunction. These mechanisms may be different from pQRSd in patients with HF, which seems to be a permanent change associated with LV dysfunction.

We described 2 representative cases of $\mathrm{pQRSd}$. In the first case (Figure 2A), QRS duration was normalized in a few days and the female patient was discharged ambulatory. In contrast, in the second case (Figure 2B), QRS duration was not normalized, and the male patient died despite intensive treatment. In this study, 4 of 5 patients with persistent $\mathrm{pQRSd}$ died. Although we did not analyze it statistically because of the small number of patients, persistent $\mathrm{pQRSd}$ may be associated with more serious complications. A further study should be conducted to clarify the clinical differences between transient and persistent pQRSd in TC.

On ROC analysis, the best cut-off point of QRS duration was $110 \mathrm{~ms}$ in both in-hospital death and cardiac death. This indicates that mild pQRSd may be a sign of myocardial damage in patients with TC. We may have to prepare for 
severe complications even when QRS prolongation is in the mild range (i.e. $100-120 \mathrm{~ms}$ ).

\section{Study Limitations}

Several study limitations should be acknowledged. First, this was a retrospective, multicenter, observational study, and there were some missing or unavailable data. For example, in LVEF, we analyzed only $61.9 \%$ of patients (185/299). LVEF was reported as an independent predictor of in-hospital outcome, ${ }^{23}$ but LVEF could not be used in multivariate logistic regression analysis because of many missing data points. Similarly, we could not examine the significance of LV outflow tract obstruction on multivariate analysis. Therefore, these may be residual confounding factors. Nevertheless, we used variables such as shock on arrival, $\mathrm{HF}$ on arrival, and BNP, which seemed to reflect severity.

Second, we had data only on in-hospital mortality, therefore the clinical impact of pQRSd on long-term outcome is unclear, and hence, we will be carrying out additional studies of long-term outcome.

Third, the first ECG was evaluated at patient admission, therefore ECG changes at the true time of onset could not be determined. In this study, however, ST-segment elevation was noted in $75.3 \%(225 / 299)$ of patients, indicating that most of them were admitted to hospital at a relatively earlier stage after onset of symptoms, compared with a recently reported large multicenter registry in which ST-segment elevation was noted in $43.7 \% .5$ In this setting, Tokyo has a special system called the CCU-HOTLINE to convey patients with suspected cardiovascular disease directly to professional facilities as soon as possible. Thus, these data may precisely reflect QRS duration at the onset of symptoms.

Fourth, we could not obtain original copies of ECG at discharge for patients who had been admitted without pQRSd in 2010, or serial ECG during the hospital course in all cases; therefore, we could not exclude the possibility that QRS duration on admission was within the normal limit but was prolonged after hospitalization in some cases, which may affect the prognosis. In the present study, however, no patients had normal QRS duration at admission and pQRSd at discharge in 2011 and 2012.

Fifth, in 5 patients with pQRSd (14.7\%), ECG at discharge showed pQRSd. In these patients, we were not able to distinguish between stress-induced and pre-existing pQRSd. In a previous report, however, bundle branch block was noted in only $0.18 \%$ of the general population, ${ }^{24}$ therefore the prevalence of pre-existing bundle branch block in the present study would have been very small. In addition, the extremely high mortality rate in these 5 patients $(4 / 5$ patients died) suggests stress-induced rather than pre-existing pQRSd. Given that we are often unable to obtain ECG before admission in daily practice, we believe that the present results highlight the clinical importance of $\mathrm{pQRSd}$ at admission for predicting critical events.

Last, the study sample size may be another possible limitation. Although we carefully selected 299 patients for enrolment, this is relatively smaller than the cohorts of previous multicenter registries.

\section{Conclusions}

PQRSd at admission in patients with TC was significantly associated with poor clinical outcome. Aggressive intervention may be required to prevent severe complications.

\section{Acknowledgments}

We would like to thank Editage for help in editing and preparing the manuscript for publication.

\section{Disclosures}

The authors declare no conflicts of interest.

\section{Funding Sources}

This study was not supported by any company, grant, or fund.

The Tokyo CCU network data registry is financially supported by the Tokyo Metropolitan Government.

\section{References}

1. Sharkey SW, Maron BJ. Epidemiology and clinical profile of Takotsubo cardiomyopathy. Circ J 2014; 78: 2119-2128.

2. Tsuchihashi K, Ueshima K, Uchida T, Oh-mura N, Kimura K, Owa M, et al. Transient left ventricular apical ballooning without coronary artery stenosis: A novel heart syndrome mimicking acute myocardial infarction: Angina Pectoris-Myocardial Infarction Investigators in Japan. J Am Coll Cardiol 2001; 38: 11-18.

3. Kurisu S, Sato H, Kawagoe T, Ishihara M, Shimatani Y, Nishioka K, et al. Tako-tsubo-like left ventricular dysfunction with ST-segment syndrome mimicking acute myocardial infarction. Am Heart J 2002; 143: 448-455.

4. Adriaenssens BF, Dens JA. Apical ballooning of the left ventricle: First series in white patients. Heart 2003; 89: 1027-1031.

5. Templin C, Ghadri JR, Diekmann J, Napp LC, Bataiosu DR, Jaguszewski M, et al. Clinical features and outcomes of Takotsubo (stress) cardiomyopathy. N Engl J Med 2015; 373: 929-938.

6. Shimizu M, Nishizaki M, Yamawake N, Fujii H, Sakurada H, Isobe $\mathbf{M}$, et al. J wave and fragmented QRS formation during the hyperacute phase in takotsubo cardiomyopathy. Circ J 2014; 78: 943-949.

7. Syed FF, Asirvatham SJ, Francis J. Arrhythmia occurrence with takotsubo cardiomyopathy: A literature review. Europace 2011; 13: $780-788$.

8. Schinkel AF, Elhendy A, van Domburg RT, Biagini E, Rizzello $\mathrm{V}$, Veltman CE, et al. Prognostic significance of QRS duration in patients with suspected coronary artery disease referred for noninvasive evaluation of myocardial ischemia. Am J Cardiol 2009; 104: $1490-1493$.

9. Yamada T, Shimonagata T, Misaki N, Asai M, Makino N, Kioka H, et al. Usefulness of spatial dispersion of QRS duration in predicting mortality in patients with mild to moderate chronic heart failure. Am J Cardiol 2004; 94: 960-963.

10. Mahfoud F, Gärtner B, Kindermann M, Ukena C, Gadomski K, Klingel K, et al. Virus serology in patients with suspected myocarditis: Utility or futility? Eur J Hear Fail 2011; 13: 398-405.

11. Miyachi H, Takagi A, Miyauchi K, Yamasaki M, Tanaka H, Yoshikawa M, et al. Current characteristics and management of ST elevation and non-ST elevation myocardial infarction in the Tokyo metropolitan area: From the Tokyo CCU network registered cohort. Heart Vessels 2016; 31: 1740-1751.

12. Prasad A, Lerman A, Rihal CS. Apical ballooning syndrome (tako-tsubo or stress cardiomyopathy): A mimic of acute myocardial infarction. Am Heart $J$ 2008; 155: 408-417.

13. Killip T 3rd, Kimball JT. Treatment of myocardial infarction in a coronary care unit. A two year experience with 250 patients. Am J Cardiol 1967; 20: 457-464.

14. Matsuo S, Imai E, Horio M, Yasuda Y, Tomita K, Nitta K, et al; Collaborators developing the Japanese equation for estimated GFR. Revised equations for estimated GFR from serum creatinine in Japan. Am J Kidney Dis 2009; 53: 982-992.

15. Isogai $T$, Yasunaga $H$, Matsui $H$, Tanaka $H$, Ueda $T$, Horiguchi $\mathrm{H}$, et al. Out-of-hospital versus in-hospital takotsubo cardiomyopathy: Analysis of 3719 patients in the Diagnosis Procedure Combination database in Japan. Int J Cardiol 2014; 176: 413417.

16. Cantor AA, Goldfarb B, Ilia R. QRS prolongation: A sensitive marker of ischemia during percutaneous transluminal coronary angioplasty. Catheter Cardiovasc Interv 2000; 50: 177-183.

17. Gilmour RF Jr, Evans JJ, Zipes DP. Purkinje-muscle coupling and endocardial response to hyperkalemia, hypoxia, and acidosis. Am J Physiol 1984; 247: H303-H311.

18. Michaelides AP, Boudoulas H, Antonakoudis H, Vyssoulis GP, Toutouzas PK. Effect of a number of coronary arteries signifi- 
cantly narrowed and status of intraventricular conduction on exercise-induced QRS prolongation in coronary artery disease. Am J Cardiol 1992; 70: 1487-1489.

19. Michaelides AP, Boudoulas H, Vyssoulis GP, Skouros C, Toutouzas PK. Exercise-induced S-wave prolongation in anterior descending coronary artery stenosis. Am J Cardiol 1992; 70: $1407-1411$.

20. Morimoto S, Kato S, Hiramitsu S, Uemura A, Ohtsuki M, Kato $\mathrm{Y}$, et al. Role of myocardial interstitial edema in conduction disturbances in acute myocarditis. Heart Vessels 2006; 21: 356 360.

21. Hung Y, Lin WH, Lin CS, Cheng SM, Tsai TN, Yang SP, et al. The prognostic role of QTc interval in acute myocarditis. Acta
Cardiol Sin 2016; 32: 223-230.

22. Kurisu S, Ishibashi K, Kato Y, Mitsuba N, Dohi Y, Nishioka K, et al. Tako-tsubo cardiomyopathy complicated by QRS prolongation. Intern Med 2012; 51: 291-294.

23. Citro R, Rigo F, D'Andrea A, Ciampi Q, Parodi G, Provenza G, et al; Tako-Tsubo Italian Network Investigators. Echocardiographic correlates of acute heart failure, cardiogenic shock, and in-hospital mortality in tako-tsubo cardiomyopathy. JACC Cardiovasc Imaging 2014; 7: 119-129.

24. JCS Joint Working Group. Guidelines for risks and prevention of sudden cardiac death (JCS 2010): Digest version. Circ J 2012; 76: $489-507$. 